\title{
Erratum:
}

\section{The connection between vortex-like topological excitations and conventional excitations in quantum ferromagnetic spin systems on two-dimensional lattice and their stability}

\author{
[Int. J. Mod. Phys. B, Vol. 29, 1550209 (2015) (22 pages)] \\ Subhajit Sarkar*, Ranjan Chaudhury ${ }^{\dagger}$ and Samir K. Paul ${ }^{\ddagger}$ \\ S. N. Bose National Centre for Basic Sciences, \\ Block-JD, Sector-III, Salt Lake, Kolkata 700098, India \\ *subhajit@bose.res.in \\ †ranjan@bose.res.in \\ †smr@bose.res.in
}

Published 24 November 2015

In page 5 equation (4) viz., $\varepsilon_{0}=-(N / 2) \lambda J \hbar^{2}$ should be read as $\varepsilon_{0}=-(\mathcal{N} / 2) \lambda J \hbar^{2}$. In page 11 in the equations (18) and (19) the symbol $N$ should be $\operatorname{read}$ as $\mathcal{N}$. 